\title{
Feasibility Conditions on the Parameters of a Strongly Regular Graph
}

\author{
Vasco Moço Mano ${ }^{1,2}$ \\ Department of Mathematics \\ University of Oporto \\ Oporto, Portugal \\ Enide Andrade Martins ${ }^{3}$ \\ Department of Mathematics \\ University of Aveiro \\ Aveiro, Portugal
Luís António de Almeida Vieira ${ }^{4}$
Department of Civil Engineering
University of Oporto
Porto, Portugal

\begin{abstract}
We consider a strongly regular graph, $G$, and associate a three dimensional Euclidean Jordan algebra, $\mathcal{V}$, to the adjacency matrix $A$ of $G$. Then, by considering convergent series of Hadamard powers of the idempotents of the unique complete system of orthogonal idempotents of $\mathcal{V}$, we establish new feasibility conditions for the existence of strongly regular graphs.
\end{abstract}

Keywords: Strongly regular graph, Euclidean Jordan algebra, Matrix analysis. 


\section{Introduction}

Strongly regular graphs are a relatively new class of graphs firstly introduced in a 1963 paper by R. C. Bose, entitled Strongly regular graphs, partial geometries and partially balanced designs, [1]. One of the main problems on the study of these graphs is to find suitable feasibility conditions over their parameters. The most used and not trivial feasibility conditions are the Krein conditions and the absolute bounds (see, for instance, [2]). In this work we explore the relationship of a three dimensional Euclidean Jordan algebra with the adjacency matrix of a strongly regular graph, in order to obtain some new inequalities for the existence of strongly regular graphs.

The concept of Euclidean Jordan algebra was introduced in 1934 by Pascual Jordan, John von Neumann and Eugene Wigner in the paper On an algebraic generalization of the quantum mechanical formalism [3]. This concept has had such a wide range of applications. For instance there are applications to the theory of statistics (see [4]), to interior point methods (see [5]) and to combinatorics (see [6]). Detailed literature on Euclidean Jordan algebras can be found in the monograph by Faraut and Korányi, [7].

\section{Euclidean Jordan algebras}

In this section we present relevant concepts for our work which can be seen, for instance in [7]. Let $\mathcal{V}$ be a real vector space with finite dimension and a bilinear mapping $(u, v) \mapsto u \bullet v$. If for all $u$ and $v$ in $\mathcal{V}$ we have $u \bullet v=v \bullet u$ and $u \bullet\left(u^{2} \bullet v\right)=u^{2} \bullet(u \bullet v)$, with $u^{2}=u \bullet u$, then $\mathcal{V}$ is called a Jordan algebra. If $\mathcal{V}$ contains an element, $e$, such that for all $u$ in $\mathcal{V}$ we have $e \bullet u=u \bullet e=$ $u$, then $e$ is called the unit element of $\mathcal{V}$. Given a Jordan algebra $\mathcal{V}$ with unit element, if there is an inner product $\langle\cdot, \cdot\rangle$ that verifies the equality $\langle u \bullet v, w\rangle=\langle v, u \bullet w\rangle$, for any $u, v, w$ in $\mathcal{V}$, then $\mathcal{V}$ is called an Euclidean Jordan algebra. In an Euclidean Jordan algebra $\mathcal{V}$, with unit element, if there is $c \in \mathcal{V}$ such that $c^{2}=c$, then $c$ is called idempotent. Two idempotents $c$ and $d$ are orthogonal if $c \bullet d=0$. A set $\left\{c_{1}, c_{2}, \ldots, c_{k}\right\}$ is called a complete system of orthogonal idempotents if $c_{i}^{2}=c_{i}, \forall i \in\{1, \ldots, k\}, c_{i} \bullet c_{j}=0, \forall i \neq j$ and $c_{1}+c_{2}+\cdots+c_{k}=e$

1 This research was supported by Center of Research of Mathematics of University of Porto from Fundação para a Ciência e Tecnologia - FCT.

2 Email: vascomocomano@gmail.com

3 Email: enide@ua.pt

4 Email: lvieira@fe.up.pt 
Let $\mathcal{V}$ be an Euclidean Jordan algebra with unit element $e$ and $u$ in $\mathcal{V}$. Then, there exist unique distinct real numbers $\lambda_{1}, \lambda_{2}, \ldots, \lambda_{k}$, and a unique complete system of orthogonal idempotents $\left\{c_{1}, c_{2}, \ldots, c_{k}\right\}$ such that

$$
u=\lambda_{1} c_{1}+\lambda_{2} c_{2}+\cdots+\lambda_{k} c_{k},
$$

with $c_{j}, j=1, \ldots, k$, real numbers (see [7], Theorem III.1.1). The $\lambda_{j}$ 's are the eigenvalues of $u$ and the decomposition (1) is the spectral decomposition of $u$.

\section{Strongly regular graphs}

In this text we consider non-empty, simple graphs and not complete graphs, herein called graphs.

Let $G$ be a graph of order $n$. Then $G$ is a $(n, k, a, c)$-strongly regular graph if it is $k$-regular and any pair of adjacent vertices have $a$ common neighbors and any pair of non-adjacent vertices have $c$ common neighbors. The parameters of a $(n, k, a, c)$-strongly regular graph are not independent and are related by the equality $k(k-a-1)=(n-k-1) c$. This equation is an example of a feasibility condition that must be satisfied by the parameters of any strongly regular graph. There are many other feasibility conditions over the parameters of a strongly regular graph. Among the most important feasibility conditions there are the Krein Conditions obtained in 1973 by Scott, Jr., [8]. However, there are still many parameter sets for which we do not know if they correspond to a strongly regular graph. In this work we deduce some new conditions to claim the unfeasibility of certain parameter sets of strongly regular graphs. We are able to deduce them by associating an Euclidean Jordan algebra to the adjacency matrix of a strongly regular graph.

\section{Associating an Euclidean Jordan algebra to a strongly regular graph}

Let $G$ be a $(n, k, a, c)$-strongly regular graph such that $0<c<k<n-1$ and $A$ be the adjacency matrix of $G$ with three distinct eigenvalues, namely $k, \theta$, and $\tau$. Here $k$ and $\theta$ are the positive eigenvalues and $\tau$ is the negative eigenvalue of $A$. Now we consider the Euclidean Jordan subalgebra of $\mathcal{V}$ spanned by $I_{n}$ and the natural powers of $A$. Since $A$ has three distinct eigenvalues, then $\mathcal{V}$ is a three dimensional Euclidean Jordan algebra.

Let $\mathcal{B}=\left\{E_{1}, E_{2}, E_{3}\right\}$ be the unique complete system of orthogonal idempotents of $\mathcal{V}$ associated to $A$, with $E_{1}=I_{n} / n+A / n+\left(J_{n}-A-I_{n}\right) / n$, $E_{2}=(-\tau n+\tau-k) I_{n} /(n(\theta-\tau))+(n+\tau-k) A /(n(\theta-\tau))+(\tau-k)\left(J_{n}-\right.$ 
$\left.A-I_{n}\right) /(n(\theta-\tau)), E_{3}=(\theta n+k-\theta) I_{n} /(n(\theta-\tau))+(-n+k-\theta) A /(n(\theta-$ $\tau))+(k-\theta)\left(J_{n}-A-I_{n}\right) /(n(\theta-\tau))$, where $J_{n}$ is the matrix whose entries are all equal to 1 and $I_{n}$ is the identity matrix of order $n$.

Now let $p$ be a natural number such that $p \geq 2$ and denote by $M_{n}(\mathbb{R})$ the set of square matrices of order $n$ with real entries. For $B$ in $M_{n}(\mathbb{R})$, we denote by $B^{\circ p}$ and $B^{\otimes p}$ the Hadamard power and the Kronecker power of order $p$ of $B$, respectively, with $B^{\circ 1}=B, B^{\circ 0}=J_{n}$ and $B^{\otimes 1}=B$. Here, we introduce a compact notation for the Hadamard and the Kronecker powers of the elements of $S$. Let $x$ and $\alpha$ be the integers such that $1 \leq \alpha \leq 3$ and $x \geq 0$. Then, we consider $E_{\alpha}^{\circ x}=\left(E_{\alpha}\right)^{\circ x}$ and $E_{\alpha}^{\otimes x}=\left(E_{\alpha}\right)^{\otimes x}$.

\section{$5 \quad$ New feasibility conditions for strongly regular graphs}

Consider the following spectral decomposition of $A, A=k E_{1}+\theta E_{2}+\tau E_{3}$. For $l \in \mathbb{N}$, let:

$$
S_{3(2 l-1)}^{\otimes}=E_{3} \otimes J_{n}^{\otimes(2 l-2)}+E_{3}^{\otimes 3} \otimes J_{n}^{\otimes(2 l-4)}+\cdots+E_{3}^{\otimes(2 l-1)},
$$

where each summand is a Kronecker product with $2 l-1$ factors. The sum $S_{3(2 l-1)}^{\otimes}$ has a principal submatrix given by:

$$
S_{3(2 l-1)}^{\circ}=E_{3} \circ J_{n}^{\circ(2 l-2)}+E_{3}^{\circ 3} \circ J_{n}^{\circ(2 l-4)}+\cdots+E_{3}^{\circ(2 l-1)}
$$

Observe that $S_{3(2 l-1)}^{\circ}=\sum_{i=1}^{l} E_{3}^{\circ(2 i-1)}$. Let $q_{3(2 l-1)}^{1}, q_{3(2 l-1)}^{2}$ and $q_{3(2 l-1)}^{3}$ be the real numbers such that $S_{3(2 l-1)}^{\circ}=\sum_{i=1}^{3} q_{3(2 l-1)}^{i} E_{i}$. Since the set $\mathcal{C}=$ $\left\{E_{i_{1}} \otimes E_{i_{2}} \otimes \cdots \otimes E_{i_{2 l-1}}: i_{1}, i_{2}, \cdots, i_{2 l-1} \in\{1,2,3\}\right\}$ is a complete system of orthogonal idempotents that is a basis of the real Euclidean Jordan algebra $\mathcal{V}^{\otimes(2 l-1)}$ spanned by $I^{\otimes(2 l-1)}$ and the natural powers of $A^{\otimes(2 l-1)}$, then the minimal polynomial of $S_{3(2 l-1)}^{\otimes}$ is $f(\lambda)=(\lambda-0) \prod_{i=1}^{l}\left(\lambda-n^{2(l-i)}\right)$. Note that, to obtain the minimal polynomial, we use the system of orthogonal idempotents in each summand of (2) (see [7, p. 44]) The matrix in (3) is a principal submatrix of $S_{3(2 l-1)}^{\otimes}$ and $f$ is the minimal polynomial of $S_{3(2 l-1)}^{\otimes}$. By interlacing Theorem (see [9, Theorem 4.3.15]), the eigenvalues of $S_{3(2 l-1)}^{\circ}$ are all nonnegative.

Since $\theta-\tau>1$ and $|\tau|>1$, then

$$
\left|\frac{\theta n+k-\theta}{n(\theta-\tau)}\right|<1,\left|\frac{-n+k-\theta}{n(\theta-\tau)}\right|<1 \text { and }\left|\frac{k-\theta}{n(\theta-\tau)}\right|<1 \text {. }
$$

Therefore, the series $\sum_{i=1}^{+\infty} E_{3}^{\circ(2 i-1)}$ is convergent with sum $s_{3}$. Consider the real numbers $q_{3 \infty}^{1}, q_{3 \infty}^{2}, q_{3 \infty}^{3}$ such that 
(4) $s_{3}=\lim _{n \rightarrow+\infty} S_{3(2 l-1)}^{\circ}=q_{3 \infty}^{1} E_{1}+q_{3 \infty}^{2} E_{2}+q_{3 \infty}^{3} E_{3}$.

As

(5) $S_{3(2 l-1)}^{\circ}=q_{3(2 l-1)}^{1} E_{1}+q_{3(2 l-1)}^{2} E_{2}+q_{3(2 l-1)}^{3} E_{3}$.

applying limits to (5) and comparing expressions (4) and (5) we obtain $q_{3 \infty}^{1}=$ $\lim _{l \rightarrow \infty} q_{3(2 l-1)}^{1}, q_{3 \infty}^{2}=\lim _{l \rightarrow \infty} q_{3(2 l-1)}^{2}, q_{3 \infty}^{3}=\lim _{l \rightarrow \infty} q_{3(2 l-1)}^{3}$. As the eigenvalues of $S_{3(2 l-1)}^{\circ}$ are nonnegative, it follows that $q_{3 \infty}^{1} \geq 0, q_{3 \infty}^{2} \geq 0$ and $q_{3 \infty}^{3} \geq 0$. Then from identity (??) and doing some algebraic manipulations we obtain:

$$
\begin{aligned}
q_{3 \infty}^{1} & =\frac{n(\theta-\tau)(n \theta-\theta+k)}{n^{2}(\theta-\tau)^{2}-(n \theta-\theta+k)^{2}}+\frac{n(\theta-\tau)(-n+k-\theta)}{n^{2}(\theta-\tau)^{2}-(-n+k-\theta)^{2}} k+ \\
& +\frac{n(\theta-\tau)(k-\theta)}{n^{2}(\theta-\tau)^{2}-(k-\theta)^{2}}(n-k-1) .
\end{aligned}
$$

The other real numbers $q_{3 \infty}^{2}$ and $q_{3 \infty}^{3}$ are obtained with similar arguments. Now, since $\lambda_{\min }(A \circ B) \geq \lambda_{\min }(A) \lambda_{\min }(B)$ for any matrices $A, B \in \mathcal{M}_{n}(\mathbb{R})$, (see $\left[10\right.$, p. 312]), and the parameters $q_{3 \infty}^{1}, q_{3 \infty}^{2}$ and $q_{3 \infty}^{3}$ are nonnegative we then the eigenvalues of $s_{3}^{\circ x}$ are also nonnegative, fot $x \in \mathbb{N}$. Let $q_{x s_{3}}^{i}$, for $i \in\{1,2,3\}$, be the real numbers such that $s_{3}^{\circ x}=\sum_{i=1}^{3} q_{x s_{3}}^{i} E_{i}$. Analyzing the parameters $q_{x s_{3}}^{1}$ and $q_{x s_{3}}^{3}$ and after some algebraic manipulation we establish the following theorem that is a new feasibility condition for the existence of strongly regular graphs.

Theorem 5.1 Let $G$ be $a(n, k, a, c)$-strongly regular graph, such that $0<c<$ $k<n-1$, whose adjacency matrix has the eigenvalues $k, \theta$ and $\tau$. Let $x \in \mathbb{N}$, then

(6) $0 \leq\left(\frac{n(\theta-\tau)(n \theta-\theta+k)}{n^{2}(\theta-\tau)^{2}-(n \theta-\theta+k)^{2}}\right)^{2 x-1}$

$$
\begin{aligned}
& +\left(\frac{n(\theta-\tau)(-n+k-\theta)}{n^{2}(\theta-\tau)^{2}-(-n+k-\theta)^{2}}\right)^{2 x-1} k+ \\
& +\left(\frac{n(\theta-\tau)(k-\theta)}{n^{2}(\theta-\tau)^{2}-(k-\theta)^{2}}\right)^{2 x-1}(n-k-1), \\
\text { (7) } 0 \leq & \left.\leq \frac{n(\theta-\tau)(n \theta-\theta+k)}{n^{2}(\theta-\tau)^{2}-(n \theta-\theta+k)^{2}}\right)^{2 x}+ \\
& +\left(\frac{n(\theta-\tau)(-n+k-\theta)}{n^{2}(\theta-\tau)^{2}-(-n+k-\theta)^{2}}\right)^{2 x} \tau+ \\
& +\left(\frac{n(\theta-\tau)(k-\theta)}{n^{2}(\theta-\tau)^{2}-(k-\theta)^{2}}\right)^{2 x}(-\tau-1) .
\end{aligned}
$$


The following result is obtained from inequality (7) of Theorem 5.1.

Theorem 5.2 Let $G$ be $a(n, k, a, c)$-strongly regular graph, such that $0<c<$ $k<n-1$, whose adjacency matrix has the eigenvalues $k, \theta$ and $\tau$. If $k<n / 2$, then

$$
(-2 \tau-1)^{2}(4 \theta-2 \tau+1)^{2}|\tau| \leq \frac{4 n}{n-2 k}(2 \theta+1)^{2}(\theta-\tau)^{4} .
$$

For a fixed $n, k$ and $\theta$ and analysing inequality (8) we observe that the left hand side is a polynomial in $\tau$ of degree 5 , with positive coefficients and the right hand side is a polynomial in $\tau$ of degree 4 with positive coefficients. Therefore one may conclude that it $|\tau|$ is bigger than $\theta$, then $|\tau|$ cannot be too large.

\section{Conclusions and Experimental Results}

In this section we present some examples of parameter sets $(n, k, a, c)$ that do not verify the equality (6) and (7). In Table 1 we consider the parameter sets $P_{1}=(201,100,2,97), P_{2}=(1585,784,33,735), P_{3}=(23989,11988,987,10989)$ and $P_{4}=(19999001,9999000,8999,9989001)$. For each set we present the respective eigenvalues $\theta$ and $\tau$, and the parameters $q_{(2 y+1)_{s_{3}}}^{1}$ and $q_{(2 y) s_{3}}^{3}$ for some values of $y$. We also consider $q_{\theta \tau k n}=\frac{4 n}{n-2 k}(2 \theta+1)^{2}(\theta-\tau)^{4}-(-2 \tau-1)^{2}(4 \theta-$ $2 \tau+1)^{2}|\tau|$.

\begin{tabular}{|c||c|c|c|c|}
\hline Parameters & $P_{1}$ & $P_{2}$ & $P_{3}$ & $P_{4}$ \\
\hline \hline$\theta$ & 0.032 & 0.07 & 0,1 & 0.001 \\
\hline$\tau$ & -95 & -702 & -10002 & $-1.0 \times 10^{7}$ \\
\hline$q_{3 s_{3}}^{1}$ & $-2.9 \times 10^{-7}$ & $-1.2 \times 10^{-8}$ & $-3.2 \times 10^{-12}$ & $-2.5 \times 10^{-19}$ \\
\hline$q_{2 s_{3}}^{3}$ & $-5.2 \times 10^{-5}$ & $-1.5 \times 10^{-5}$ & $-5.4 \times 10^{-8}$ & $-5.0 \times 10^{-12}$ \\
\hline$q_{5 s_{3}}^{1}$ & $-1.6 \times 10^{-11}$ & $-1.2 \times 10^{-14}$ & $-1.6 \times 10^{-20}$ & $-1.3 \times 10^{-33}$ \\
\hline$q_{6 s_{3}}^{3}$ & $-1.2 \times 10^{-13}$ & $-1.2 \times 10^{-17}$ & $-1.0 \times 10^{-24}$ & $-9.5 \times 10^{-41}$ \\
\hline$q_{\theta \tau k n}$ & $-5.0 \times 10^{10}$ & $-2.6 \times 10^{15}$ & $-1.5 \times 10^{21}$ & $-1.6 \times 10^{36}$ \\
\hline
\end{tabular}

Table 1

Numerical results for $P_{1}, P_{2}, P_{3}$ and $P_{4}$.

From the data presented in Table 1 we confirm the results expressed in Theorem 5.2, namely we confirm that if $\theta$ is smaller than $|\tau|$, then we conclude 
that the sequence $(n, k, a, c)$ does not correspond to a parameter set of a strongly regular graph.

\section{References}

[1] Bose, R. C. Strongly regular graphs, partial geometries and partially balanced designs, Pacific J. Math 13 (1963), 389-419.

[2] Godsil, C., and G. Royle, "Algebraic Graph Theory", Springer, 2001.

[3] Jordan, P., J. v. Neuman, and E. Wigner, On an algebraic generalization of the quantum mechanical formalism, Annals of Mathematics, 35 (1934), 29-64.

[4] Massan, H., and E. Neher, Estimation and testing for lattice conditional independence models on Euclidean Jordan algebras, Ann. Statist., 26 (1998), 1051-1082.

[5] Faybusovich, L., Linear systems in Jordan algebras and primal-dual interiorpoint algorithms, Journal of Computational and Applied Mathematics, 86 (1997), 148-175.

[6] Cardoso, D. M., and L. A. Vieira, Euclidean Jordan Algebras with Strongly Regular Graphs, Journal of Mathematical Sciences, 120 (2004), 881-894.

[7] Faraut, J., and A. Korányi, "Analysis on Symmetric Cones", Oxford Science Publications, 1994.

[8] Scott, Jr. L. L. A condition on Higman's parameters, Notices of Amer. Math. Soc., 20 A-97 (1973), 721-20-45.

[9] Horn, R., and C. R. Johnson, "Matrix Analysis", Cambridge University Press, 1985.

[10] Horn, R., and C. R. Johnson, "Topics in Matrix Analysis", Cambridge University Press, 1991. 